\title{
A Parameterized Model of Bolted Joints in Machine Tools
}

\author{
Hongqi $\mathrm{Li}$ and $\mathrm{Bin} \mathrm{Li}$ \\ National NC System Engineering Research Center, Huazhong University of Science and Technology, Wuhan, \\ 430074, China
}

\section{Kuanmin Mao and Xiaolei Huang}

State Key Laboratory of Digital Manufacturing Equipment and Technology, Huazhong University of Science and Technology, Wuhan, 430074, China

\author{
Fangyu Peng \\ National NC System Engineering Research Center, Huazhong University of Science and Technology, Wuhan, \\ 430074, China
}

(Received 7 July 2012; provisionally accepted 4 April 2013; accepted 21 October 2013)

\begin{abstract}
A new dynamic model for bolted joints in heavy machine structures is proposed. The influence of the bolt pretightening force, the interface dimensions and the surface roughness on joint dynamics (i.e. the stiffness matrix of finite element) were analysed. Each stiffness coefficient in the matrix can be expressed by an exponential function of preload, and a quadratic polynomial function of geometric dimensions. Sixteen specimens were thoroughly designed and analysed. Then, the resulting stiffness matrices were saved as templates, based on which could establish dynamic models of joints with different influence factor values, using curve-fitting and Response Surface Methodology (RSM). The methodology was validated by experiments with a specimen of the new design and a heavy-duty cutting machine.
\end{abstract}

\section{INTRODUCTION}

The characteristics of joints have significant influence on the dynamic performance of whole structures. ${ }^{1}$ Research on the modelling and identification of joint stiffness has been conducted widely for machine structures. Yoshimura ${ }^{2}$ proposed a dynamic model to analyse the joints between two components using an equivalent spring-damper system of six degrees of freedom. Greenwood ${ }^{3}$ modelled the joint dynamics with a normal spring, according to the Hertz contact theory. Some other researchers ${ }^{4,5}$ modelled the joint dynamics with a group of spring-dampers. Based on the Least Square Method, Tsai and Chou, ${ }^{6}$ as well as Wang and Ren et al., ${ }^{7,8}$ extracted the joint dynamic parameters by analysing the Frequency Response Function (FRF). Ahmadian and Jalali ${ }^{2,3}$ formulated a non-linear generic element model for bolted lap joints by satisfying all known conditions for a joint interface, and hence provided a non-linear parametric formulation for the families of allowable joint models. According to the stiffness influence factor, a novel dynamic model of fixed joints was proposed by K. Mao and B. Li, ${ }^{9}$ which elaborated the identification of the model parameters in detail and verified its high accuracy through experiments.

Based on the study of the influence factors of joint dynamics, this paper presents a parameterized dynamic model of bolted joints, whose parameters can be calculated without additional experiments as long as the values of the involved influence factors (e.g., preloads, interface dimensions, and surface roughness) are known. Therefore, this studymay be widely applied in industry.

This paper is organized as follows: Section 2 describes the

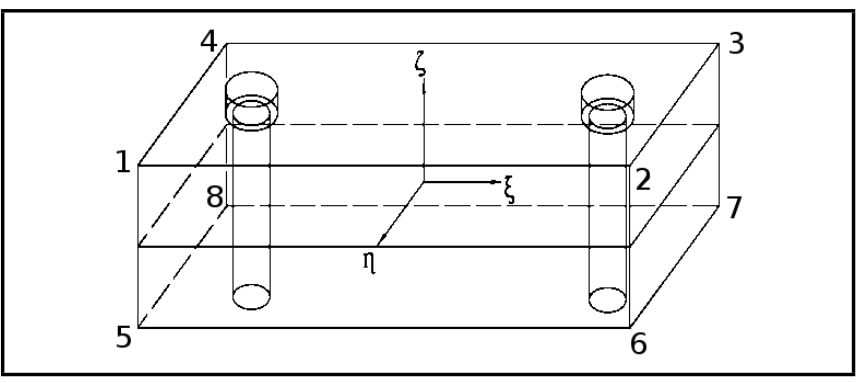

Figure 1: The bolted joint element.

effects of the preloads, the geometric dimensions, and the surface roughness on bolted joint dynamics. The sample database of joints is described in Section 3, with a detailed modelling process. In Section 4, the proposed model is experimentally validated with designed specimen and a heavy-duty cutting machine.

\section{ANALYSIS OF THE EFFECT OF BOLTED- JOINT DYNAMICS}

\subsection{Introduction to the Influence Factors}

Based on a wide investigation of manufacturing shops, a new model of bolted joint element with eight nodes was proposed by K. Mao et al. ${ }^{9}$ as shown in Fig. 1.

According to the definition of the stiffness influence factor, the model of the bolted joint element can be expressed as in Eq. 1, where $i, m=1,2,3,4$ represent the node; $j, n=1,2,3$ represent the direction; and $K_{m n}^{i j}$ is the stiffness influence coefficient. This correspond to the necessary force imposed on 